\title{
Helicobacter pylori no idoso
}

Luiz Gonzaga Vaz Coelho

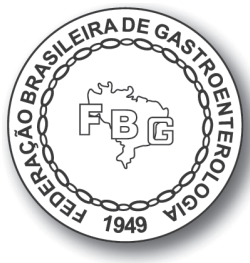

Maria Clara Freitas Coelho

\section{FUNÇÃO GÁSTRICA E ENVELHECIMENTO}

O estômago sadio não é marcadamente alterado pelo envelhecimento. A função motora é bem preservada, com modesto retardo do esvaziamento gástrico $^{1}$. Pode ocorrer, entretanto, pequeno comprometimento do fluxo sanguíneo para a mucosa gástrica com mínima redução na produção de muco, bicarbonato e prostaglandinas, o que pode contribuir para o surgimento de queixas dispépticas, DRGE e lesões induzidas por anti-inflamatórios não hormonais $(\mathrm{AINH})^{2,3}$. Hoje, está bem estabelecido que, em um estômago sadio, a secreção ácida é mantida ao longo da vida, com leve redução na produção de pepsina ${ }^{4,5}$.

A identificação do H. pylori ${ }^{6}$ e os estudos que se seguiram mostraram seu inquestionável papel como principal agente causal das gastrites crônicas (antes consideradas como secundárias ao processo de envelhecimento) e também nas sequelas tardias da infecção como a úlcera péptica, o adenocarcinoma e o linfoma MALT do estômago ${ }^{7}$. A infecção tem participação, também, em outras situações clínicas, como dispepsia funcional, anemia ferropriva de causa obscura, púrpura trombocitopênica idiopática, deficiência de vitamina B12, complicações associadas ao uso de ácido acetilsalicílico (AAS) e AINH e desenvolvimento de tumores gástricos metacrônicos após ressecção endoscópica ou cirúrgica de neoplasias gástricas. Finalmente, a infecção por $H$. pylori tem sido associada, positiva ou negativamente, com uma série de condições extragastroduodenais, como aterosclerose, diabetes, doenças neurológicas (AVC, doença de Alzheimer e doença de Parkinson), obesidade, asma e outros quadros atópicos. Entretanto, nessas situações, não há, ainda, evidências de plausibilidade biológica e causalidade provadas ${ }^{8-10}$. 
O objetivo deste capítulo é analisar aspectos relacionados a prevalência, afecções associadas, diagnóstico e tratamento da infecção por $H$. pylori no paciente idoso.

\section{PREVALÊNCIA DE H. PYLORI NO IDOSO}

Uma metanálise recente avaliando 183 estudos provenientes de 73 países dos 5 continentes estima uma prevalência global de infecção por H. pylori de 44,3\% (IC 95\%: 9-44,7), com taxas mais elevadas na infância, de 48,6\% (IC 95\%: 43,8-53,5), quando comparadas com a população adulta (32,6\% [IC 95\%: $28,4-36,8]$ ). Estudos realizados há 2 décadas mostravam um aumento na prevalência da infecção na população idosa, atingindo 40 a $60 \%$ dos idosos assintomáticos e mais de 70\% naqueles com afecções gastroduodenais ou institucionalizados ${ }^{11}$. De modo interessante, uma redução pronunciada na prevalência da infecção tem sido descrita em idosos acima de 85 anos. O desaparecimento da bactéria, seja secundário à progressão das lesões gástricas com consequente decréscimo nos níveis séricos circulantes de IgG ou à presença de atrofia gástrica e áreas extensas de metaplasia intestinal (MI), e o uso corrente ou prévio de antibióticos e antissecretores são os mecanismos especulados para justificar tais achados ${ }^{12-15}$. Tais estudos reforçam os cuidados para o estabelecimento correto do diagnóstico da infecção na população geriátrica.

\section{DIAGNÓSTICO DA INFECÇÃO}

O diagnóstico da infecção pode ser estabelecido por métodos não invasivos e invasivos e cursa com algumas particularidades a ser observadas no idoso.

\section{Métodos invasivos}

Compreendem a realização da endoscopia digestiva alta (EDA) e a coleta de material para realização de estudo histológico e microbiológico, além do teste da urease. A histologia é considerada o padrão-ouro na detecção da infecção pelo $H$. pylori, com a vantagem de avaliar a presença de alterações morfológicas da mucosa gástrica. Sua acurácia é também influenciada por fatores como o local e o número de biópsias, métodos de coloração, uso de inibidores da bomba protônica (IBP) e antibióticos, assim como a experiência do patologista. Duas biópsias de antro e corpo (pequena e grande curvatura) são habitualmente recomendadas ${ }^{10}$. Em algumas situações, a elevada presença de atrofia gástrica no idoso pode reduzir a sensibilidade do estudo histológico ${ }^{16}$. 
O teste da urease é um teste invasivo barato, rápido, com especificidade acima de $95 \%$ e sensibilidade variável. O número de fragmentos estudados é capaz de aumentar a sensibilidade do método. Estudos demonstram que a coleta de fragmentos de antro e corpo aumenta a acurácia do teste da urease e reduz os resultados falso-negativos em virtude da eventual baixa densidade do $\mathrm{H}$. pylori na mucosa gástrica, além de acelerar o tempo de reação do teste ${ }^{10}$. Um estudo em idosos mostrou sensibilidade menor em pacientes acima de 60 anos quando comparado com indivíduos mais jovens $(57 \% \text { vs. } 75 \%)^{17}$.

Os métodos microbiológicos, além de fornecer um diagnóstico definitivo da infecção, permitem o estudo da suscetibilidade da amostra aos antimicrobianos, bem como sua capacidade de produzir citotoxinas. Em virtude das dificuldades técnicas em sua realização, não é usada comumente na prática diária. Mais recentemente, estudos genotípicos (PCR) têm sido empregados para avaliação de resistência antimicrobiana ao H. pylori ${ }^{18}$. O uso de IBP deve ser interrompido até 2 semanas antes da realização dos testes diagnósticos da infecção pelo $H$. pylori, exceto a sorologia. O uso de antibióticos e de sais de bismuto deve ser interrompido até 4 semanas antes dos testes diagnósticos.

\section{Métodos não invasivos}

Compreendem o teste respiratório com ureia marcada com carbono 13 ou 14, a pesquisa de antígenos fecais e a sorologia. Da mesma forma que no teste da urease, a atividade ureásica constitui a base dos testes respiratórios. É hoje universalmente aceito como o método não invasivo padrão-ouro para o diagnóstico e o controle de tratamento da infecção por $H$. pylori em adultos e crianças acima de 6 anos, com sensibilidade e especificidade sempre acima de $95 \%$. Estudos realizados em idosos com o teste respiratório com ureia marcada têm demonstrado que o desempenho do teste não é afetado por potenciais covariáveis como função cognitiva, incapacidade, comorbidades e cotratamentos ${ }^{19,20}$. Embora apresente excelente acurácia, seu emprego ainda não está incorporado à prática diária no Brasil, especialmente pelas dificuldades na importação e no licenciamento do uso do substrato pelas autoridades nacionais.

O teste do antígeno fecal, particularmente com o emprego de anticorpos monoclonais, é também um teste robusto para o diagnóstico da infecção e da erradicação do $H$. pylori. Para manter sua sensibilidade, é necessário que as fezes sejam estocadas à temperatura ambiente por, no máximo, 24 horas, ou refrigeradas a $4{ }^{\circ} \mathrm{C}$ por até 72 horas. Uma limitação na população idosa é a frequente presença de constipação. $\mathrm{O}$ retardo do tempo de trânsito gastrintestinal, 
inclusive nos cólons, poderia promover a degradação dos antígenos bacterianos e comprometer sua detecção ${ }^{14}$.

Os testes sorológicos baseiam-se na detecção de anticorpos IgG anti-H. pylori e são não invasivos, amplamente disponíveis, baratos, de fácil execução e de fácil aceitação pelos pacientes. A sorologia tem como principal indicação o rastreamento da infecção por $H$. pylori em estudos epidemiológicos. Sua principal limitação é não distinguir entre infecção ativa e passada, uma vez que os anticorpos IgG anti- $H$. pylori podem persistir elevados no soro por longos períodos, mesmo após a erradicação, não servindo como método de controle de erradicação pós-tratamento. Por vezes, a sorologia pode ser útil em pacientes com pequena população bacteriana (uso de antibióticos ou antissecretores, sangramento gástrico, lesões malignas e gastrite atrófica extensa), sendo o único teste a se mostrar positivo. Pacientes idosos com imunodeficiências ou desnutrição proteica podem apresentar sorologia falso-negativa por ausência de resposta imune $e^{14,21}$.

\section{AFECÇÕES ASSOCIADAS}

\section{Gastrite crônica, atrofia gástrica e metaplasia intestinal}

A sequência carcinogenética de infecção pelo $H$. pylori $\rightarrow$ gastrite crônica $\rightarrow$ atrofia glandular $\rightarrow$ metaplasia intestinal $\rightarrow$ displasia $\rightarrow$ adenocarcinoma do tipo intestinal constitui o fundamento inicial para os estudos da carcinogênese gástrica ${ }^{22}$. A erradicação da bactéria na população idosa é capaz de reduzir a atividade da gastrite crônica quando comparada com idosos não tratados ${ }^{23}$. Estudo de seguimento de longo prazo ( 8,4 anos) envolvendo 84 pacientes idosos com gastrite atrófica intensa demonstrou que a erradicação de $H$. pylori promoveu redução da atrofia e impediu a progressão da MI.

\section{Adenocarcinoma e linfoma MALT gástricos}

Estima-se que aproximadamente $80 \%$ dos tumores gástricos malignos estejam associados com a infecção por $H$. pylori. O seguimento endoscópico é recomendado em pacientes com gastrite atrófica e MI como forma de permitir o diagnóstico precoce do câncer gástrico e melhora da sobrevida. A erradicação do H. pylori tem o potencial de diminuir o risco de câncer gástrico. Ensaios clínicos têm demonstrado que a incidência dessa neoplasia pode ser reduzida em até 30 a $40 \%$ no grupo de pacientes em que a bactéria foi erradicada ${ }^{24}$. A erradicação do H. pylori em idosos submetidos à ressecção endoscópica de tu- 
mores gástricos precoces tem o potencial de reduzir o surgimento de tumores metacrônicos no estômago remanescente ${ }^{25}$.

O linfoma MALT gástrico acomete predominantemente pacientes de meia-idade e idosos, de ambos os sexos. A infecção pelo $H$. pylori é considerada o principal fator de risco para o desenvolvimento deste linfoma, e a regressão da doença ocorre na maioria dos pacientes após a erradicação do H. pylori. Embora pouco comum, sua incidência está em elevação, tornando necessária uma compreensão abrangente de sua abordagem pelo gastroenterologista ${ }^{26}$.

\section{Úlcera péptica (ver capítulo 6 - "Doença ulceropéptica no idoso")}

\section{Afecções extradigestivas}

É bem estabelecido que condições inflamatórias crônicas podem causar repercussões em locais distantes do local da inflamação. Tem sido postulado que a infecção por H. pylori, que produz uma inflamação ativa na mucosa gástrica de longa duração, poderia estar associada a uma variedade de manifestações extradigestivas. No idoso, ganham destaque:

- Anemia por deficiência de ferro ou vitamina $B 12^{8}$.

- Distúrbio cognitivo leve e doença de Alzheimer ${ }^{27}$.

- Doença de Parkinson.

- Alterações do apetite.

- Doença coronariana e aterosclerose extracardíaca.

Com exceção da anemia por deficiência de ferro ou vitamina B12, as outras possíveis associações provêm, quase sempre, de estudos com pequeno número de participantes e com viés na seleção dos grupos-controle. Soma-se a isto uma tendência à publicação preferencial de resultados de associação positiva.

\section{TRATAMENTO}

Existem poucos estudos avaliando não só a eficácia terapêutica dos diferentes esquemas anti- $H$. pylori como também a ocorrência de efeitos adversos na população geriátrica. A Tabela 1 exibe algumas particularidades a ser observadas na abordagem terapêutica da infecção por $H$. pylori no idoso.

Habitualmente, o tratamento e o retratamento da infecção por $H$. pylori no idoso seguem as mesmas recomendações para a população geral e acham-se listados nas Tabelas 2 e 3, conforme orientação do IV Consenso Brasileiro sobre a infecção por H. pylori ${ }^{10}$. 
Tabela 1 Aspectos a ser considerados no tratamento anti-H. pylori no idoso

\begin{tabular}{ll}
\hline Resistência antibiótica & $\begin{array}{l}\text { Consumo prévio de antibacterianos, especialmente macrolídeos e } \\
\text { fluoroquinolonas }\end{array}$ \\
\hline Prescrição detalhada & $\begin{array}{l}\text { Instrução cuidadosa ao paciente ou responsável sobre o modo de } \\
\text { uso e duração do tratamento e seus possíveis efeitos adversos }\end{array}$ \\
\hline $\begin{array}{l}\text { Interação } \\
\text { medicamentosa }\end{array}$ & $\begin{array}{l}\text { Interações dos antimicrobianos com drogas comumente } \\
\text { empregadas no idoso. Polifarmácia é achado frequente }{ }^{28}\end{array}$ \\
\hline Escolha do IBP & $\begin{array}{l}\text { Menor interação medicamentosa (pantoprazol) e ação } \\
\text { independente da capacidade de metabolização do IBP pelo } \\
\text { organismo (rabeprazol e esomeprazol) }\end{array}$ \\
\hline Comor,30 & $\begin{array}{l}\text { Amoxicilina e claritromicina requerem ajuste de dose em idosos } \\
\text { com depuração de creatinina inferior a } 30 \mathrm{~mL} / \text { min }\end{array}$ \\
\hline Exame pós-tratamento & $\begin{array}{l}\text { Preferência sempre por métodos não invasivos, exceto em idosos } \\
\text { com úlcera gástrica, linfoma MALT ou gastrite intensa }\end{array}$
\end{tabular}

Tabela 2 Medicamentos, posologia e duração dos tratamentos de primeira linha da infecção por H. pylori10

\begin{tabular}{|c|c|c|c|}
\hline & Medicamento & Dose & Duração \\
\hline \multicolumn{4}{|l|}{ Recomendado } \\
\hline \multirow[t]{3}{*}{$\begin{array}{l}\text { Terapia tríplice } \\
\text { clássica }\end{array}$} & $\mathrm{IBP} *$ & $\begin{array}{l}\text { Dose plena a cada } \\
12 \mathrm{~h}\end{array}$ & \multirow[t]{3}{*}{14 dias } \\
\hline & Claritromicina & 500 mg a cada $12 \mathrm{~h}$ & \\
\hline & Amoxicilina & $1 \mathrm{~g}$ a cada $12 \mathrm{~h}$ & \\
\hline \multicolumn{4}{|l|}{ Alternativas } \\
\hline \multirow[t]{4}{*}{$\begin{array}{l}\text { Terapia quádrupla } \\
\text { com bismuto }\end{array}$} & IBP & $\begin{array}{l}\text { Dose plena a cada } \\
12 \mathrm{~h}\end{array}$ & \multirow[t]{3}{*}{10 a 14 dias } \\
\hline & $\begin{array}{l}\text { Subcitrato de bismuto } \\
\text { coloidal }\end{array}$ & $\begin{array}{l}120 \mathrm{mg} \text { a cada } 6 \mathrm{~h} \text { ou } \\
240 \mathrm{mg} \text { a cada } 12 \mathrm{~h}\end{array}$ & \\
\hline & $\begin{array}{l}\text { Cloridrato de } \\
\text { tetraciclina** }\end{array}$ & 500 mg a cada 6 h & \\
\hline & Metronidazol & 400 mg a cada 8 h & \\
\hline \multirow{4}{*}{$\begin{array}{l}\text { Terapia } \\
\text { concomitante sem } \\
\text { bismuto }\end{array}$} & IBP & $\begin{array}{l}\text { Dose plena a cada } \\
12 \mathrm{~h}\end{array}$ & \multirow[t]{4}{*}{14 dias } \\
\hline & Amoxicilina & $1 \mathrm{~g} \mathrm{a}$ cada $12 \mathrm{~h}$ & \\
\hline & Claritromicina & 500 mg a cada 12 h & \\
\hline & $\begin{array}{l}\text { Metronidazol ou } \\
\text { tinidazol }\end{array}$ & 500 mg a cada 12 h & \\
\hline
\end{tabular}

* IBP: inibidores da bomba protônica. Estudos mostram que o emprego de IBP de 2a geração (rabeprazol e esomeprazol) nesta situação parece aumentar as taxas de erradicação 9,21,25. Dose plena: omeprazol 20 mg, lansoprazol $30 \mathrm{mg}$, pantoprazol $40 \mathrm{mg}$, rabeprazol 20 mg, dexlansoprazol 60 mg, vonoprazam $20 \mathrm{mg}$ ou esomeprazol $40 \mathrm{mg}$.

** Na sua indisponibilidade, o cloridrato de tetraciclina pode ser substituído por doxiciclina $100 \mathrm{mg}$ a cada $12 \mathrm{~h}^{4}$. 
Tabela 3 Medicamentos, posologia e duração dos principais regimes recomendados como $2^{\mathrm{a}}$ ou $3^{\mathrm{a}}$ linha diante do insucesso com o esquema tríplice clássico ${ }^{10}$

\begin{tabular}{|c|c|c|c|}
\hline & Medicamento & Dose & Duração \\
\hline \multicolumn{4}{|l|}{ Recomendado } \\
\hline \multirow[t]{3}{*}{$\begin{array}{l}\text { Terapia tríplice com } \\
\text { levofloxacino }\end{array}$} & $\mathrm{IBP}^{*}$ & $\begin{array}{l}\text { Dose plena a cada } \\
12 \mathrm{~h}\end{array}$ & \multirow[t]{3}{*}{10 a 14 dias } \\
\hline & Amoxicilina & $1 \mathrm{~g}$ a cada $12 \mathrm{~h}$ & \\
\hline & Levofloxacino & $500 \mathrm{mg}$ a cada $24 \mathrm{~h}$ & \\
\hline \multirow[t]{4}{*}{$\begin{array}{l}\text { Terapia quádrupla } \\
\text { com bismuto }\end{array}$} & $\mathrm{IBP}$ & $\begin{array}{l}\text { Dose plena a cada } \\
12 \mathrm{~h}\end{array}$ & \multirow[t]{4}{*}{10 a 14 dias } \\
\hline & $\begin{array}{l}\text { Subcitrato de bismuto } \\
\text { coloidal }\end{array}$ & $\begin{array}{l}120 \mathrm{mg} \text { a cada } 6 \mathrm{~h} \text { ou } \\
240 \mathrm{mg} \text { a cada } 12 \mathrm{~h}\end{array}$ & \\
\hline & $\begin{array}{l}\text { Cloridrato de } \\
\text { tetraciclina** }\end{array}$ & 500 mg a cada 6 h & \\
\hline & Metronidazol & 400 mg a cada $8 \mathrm{~h}$ & \\
\hline
\end{tabular}

* IBP: inibidores da bomba protônica. Dose plena: omeprazol 20 mg, lansoprazol 30 mg, pantoprazol 40 mg, rabeprazol $20 \mathrm{mg}$, dexlansoprazol $60 \mathrm{mg}$, vonoprazam $20 \mathrm{mg}$ ou esomeprazol $40 \mathrm{mg}$.

** Na sua indisponibilidade, o cloridrato de tetraciclina pode ser substituído por doxiciclina $100 \mathrm{mg}$ a cada $12 h^{4}$.

Finalmente, a decisão de tratar a infecção por H. pylori também nos indivíduos idosos tem sido enfatizada. Uma coorte prospectiva recente realizada em Hong Kong analisou 73.237 indivíduos submetidos a tratamento anti- $H$. pylori entre 2003 e 2012. Acompanhados por um período mediano de 7,6 anos, 200 indivíduos $(0,27 \%)$ desenvolveram câncer gástrico. $\mathrm{O}$ risco de câncer gástrico nos indivíduos tratados foi $18 \%$ menor na população com 60 anos ou mais que haviam erradicado a bactéria há mais de 10 anos. Tais achados reforçam a indicação de tratamento do $H$. pylori em qualquer idade como medida de prevenção do câncer gástrico ${ }^{31}$.

\section{REFERÊNCIAS BIBLIOGRÁFICAS}

1. Bitar K, Greenwood-Van Meerveld B, Saad R, Wiley JW. Aging and gastrointestinal neuromuscular function: insights from within and outside the gut. Neurogastroenterol Motil. 2011;23:490-501.

2. Rayner CK, Horowitz M. Physiology of the ageing gut. Curr Opin Clin Nutr Metab Care. 2013;16:33-8.

3. Durazzo M, Campion D, Fagoonee S, Pellicano R. Gastrointestinal tract disorders in the elderly. Minerva Med. 2017;108:575-91.

4. Baron JH. Gastric secretion in a healthy man, 1949-69. Lancet. 1970;296:547-9.

5. Feldman M, Cryer B, McArthur KE, Huet BA, Lee E. Effects of aging and gastritis on gastric acid and pepsin secretion in humans: a prospective study. Gastroenterology. 1996;110:1043-52. 
6. Marshall BJ, Warren JR. Unidentified curved bacilli in the stomach of patients with gastrits and peptic ulceration. Lancet. 1984;i:1311-15.

7. Siurala M, Isokosi M, Varis K, Kekki M. Prevalence of gastritis in a rural population. Bioptic studies of subjects selected at random. Scand J Gastroent. 1968;3:211-23.

8. Malfertheiner P, Megraud F, O’Morain CA, Gisbert JP, Kuipers EJ, Axon AT, et al. Management of Helicobacter pylori infection-the Maastricht V/Florence Consensus Report. Gut. 2017;66:6-30.

9. Chey WG, Leontiadis GI, Howden CW, Moss SF. ACG Clinical Guideline: treatment of Helicobacter pylori infection. 2017;112:212-39.

10. Coelho LGV, Marinho JR, Genta R, Ribeiro LT, Passos MCF, Zaterka S, et al. IV Brazilian Consensus Conference on Helicobacter pylori infection. Arq Gastroenterol. 2018;55:97-121.

11. Pilotto A, Salles N. Helicobacter pylori infection in geriatrics. Helicobacter. 2002;7(Suppl 1):56-62.

12. Ford AC, Axon AT. Epidemiology of Helicobacter pylori infection and public health implications. Helicobacter. 2010;15(Suppl 1):1-6.

13. Neri MC, Lai L, Bonetti P, Baldassarri AR, Monti M, De Luca P, et al. Prevalence of Helicobacter pylori infection in elderly inpatients and in institutionalized old people: correlation with nutritional status. Age Ageing. 1996;25:17-21.

14. Salles-Montaudon N, Dertheil S, Broutet N, Gras N, Monteiro L, De Mascarel A, et al. Detecting Helicobacter pylori infection in hospitalized frail older patients: the challenge. J Am Geriatr Soc. 2002;50:1674-80.

15. Peleteiro B, Lunet N, Barros R, La Vecchia C, Barros H. Factors contributing to the underestimation of Helicobacter pylori-associated gastric cancer risk in a high-prevalence population. Cancer Causes Control. 2010;21:1257-64.

16. Pilotto A, Franceschi M. Helicobacter pylori infection in older people. World J Gastroenterol. 2014;20:6364-73.

17. Abdalla AM, Sordillo EM, Hanzely Z, Perez-Perez GI, Blaser MJ, Holt PR, et al. Insensitivity of the CLOtest for H. pylori, especially in the elderly. Gastroenterology. 1998;115:243-4.

18. Sanches BS, Martins GM, Lima K, Cota B, Moretzsohn LD, Ribeiro LT, et al. Detection of Helicobacter pylori resistance to clarithromycin and fluoroquinolones in Brazil: a national survey. World J Gastroenterol. 2016;22:7587-94.

19. Niv Y, Niv G, Koren R. 13C-urea breath test for diagnosis of Helicobacter pylori infection in the elderly. Dig Dis Sci. 2004;49:1840-44.

20. Atli T, Sahin S, Arslan BU, Varli M, Yalcin AE, Aras S. Comparison of the C14 urea breath test and histopathology in the diagnosis of Helicobacter pylori in the elderly. J Pak Med Assoc. 2012;62:1061-5.

21. Burns EA. Effects of aging on immune function. J Nutr Health Aging. 2004;8:9-18.

22. Correa P. Human gastric carcinogenesis: a multistep and multifactorial process - First American Cancer Society Award lecture on cancer epidemiology and prevention. Cancer Res. 1992;52:673540.

23. Pilotto A, Malfertheiner P. Review article: an approach to Helicobacter pylori infection in the elderly. Aliment Pharmacol Ther. 2002;16:683-9.

24. Herrero R, Park JY, Forman D. The fight against gastric cancer - the IARC Working Group report. Best Pract Res Clin Gastroenterol. 2014;28:1107-14.

25. Choi IJ, Kook MC, Kim YI, Cho SJ, Lee JY, Kim CG, et al. H. pylori therapy for the prevention of metachronous gastric cancer N Engl J Med. 2018;378:1085-95.

26. Juárez-Salcedo LM, Sokol L, Chavez JC, Dalia S. Primary gastric lymphoma, epidemiology, clinical diagnosis, and treatment. Cancer Control. 2018;25:1-12.

27. Doulberis M, Kotronis G, Thomann R, Polyzos SA, Boziki M, Gialamprinou D, et al. Review: impact of Helicobacter pylori on Alzheimer's disease: what do we know so far? Helicobacter. 2018;23(1). 
28. Hines LE, Murphy JE. Potentially harmful drug-drug interactions in the elderly: a review. Am J Geriatr Pharmacother. 2011;9:364-77.

29. Norgard NB, Mathews KD, Wall GC. Drug-drug interaction between clopidogrel and the proton pump inhibitors. Ann Pharmacother. 2009;43:1266-74.

30. McNicholl AG, Linares PM, Nyssen OP, Calvet X, Gisbert JP. Meta-analysis: esomeprazole or rabeprazole vs. first-generation pump inhibitors in the treatment of Helicobacter pylori infection. Aliment Pharmacol Ther. 2012;36:414-25.

31. Leung WK, Wong IO, Cheung KS, Yeung KF, Chan EW, Wong AY, et al. Effects of Helicobacter pylori treatment on incidence of gastric cancer in older individuals. Gastroenterology. 2018;155:6775 .

32. Regev A, Fraser GM, Braun M, Maoz E, Leibovici L, Niv Y. Seroprevalence of Helicobacter pylori and length of stay in a nursing home. Helicobacter. 1999;4:89-93. 